\title{
Space-charge effects in ultrahigh current electron bunches generated by laser-plasma accelerators
}

\author{
F. J. Grüner, ${ }^{1,2, *}$ C. B. Schroeder, ${ }^{3}$ A. R. Maier, ${ }^{2}$ S. Becker, ${ }^{2}$ and J. M. Mikhailova ${ }^{1,4}$ \\ ${ }^{1}$ Max-Planck Institute of Quantum Optics, 85748 Garching, Germany \\ ${ }^{2}$ Ludwig-Maximilians University of Munich, 85748 Garching, Germany \\ ${ }^{3}$ Lawrence Berkeley National Laboratory, Berkeley, California 94720, USA \\ ${ }^{4}$ Moscow State University, 119992 Moscow, Russia \\ (Received 16 June 2008; published 23 February 2009)
}

\begin{abstract}
Recent advances in laser-plasma accelerators, including the generation of GeV-scale electron bunches, enable applications such as driving a compact free-electron laser (FEL). Significant reduction in size of the FEL is facilitated by the expected ultrahigh peak beam currents (10-100 kA) generated in laser-plasma accelerators. At low electron energies such peak currents are expected to cause space-charge effects such as bunch expansion and induced energy variations along the bunch, potentially hindering the FEL process. In this paper we discuss a self-consistent approach to modeling space-charge effects for the regime of laser-plasma-accelerated ultracompact electron bunches at low or moderate energies. Analytical treatments are considered as well as point-to-point particle simulations, including the beam transport from the laser-plasma accelerator through focusing devices and the undulator. In contradiction to non-selfconsistent analyses (i.e., neglecting bunch evolution), which predict a linearly growing energy chirp, we have found the energy chirp reaches a maximum and decreases thereafter. The impact of the spacecharge induced chirp on FEL performance is discussed and possible solutions are presented.
\end{abstract}

DOI: 10.1103/PhysRevSTAB.12.020701

PACS numbers: 41.75.-i, 52.38.Kd, 41.60.Cr

\section{INTRODUCTION}

Since the demonstration in 2004 of high-quality electron beams generated by laser-plasma accelerators [1-3], there has been a growing interest in this field and the potential applications of these beams. Laser-plasma accelerated electron beams with energies up to $1 \mathrm{GeV}$ have been demonstrated experimentally in $\mathrm{cm}$-scale plasmas [4,5]. With the development of new short-pulse, high-power (petawatt) lasers, it is anticipated that $10 \mathrm{GeV}$ beams will be produced in a single plasma-accelerator stage with length $<1 \mathrm{~m}$. Plasma-based accelerators promise not only a great reduction in size (from kilometer-scale machines to centimeter-scale machines) due to the ultrahigh accelerating gradient of the plasma wave, but the electron bunches generated have intrinsically ultrashort bunch durations (a fraction of the plasma period, typically a few femtoseconds), and ultrahigh peak currents (as high as $100 \mathrm{kA}$ ). Among the most discussed applications is driving a free-electron laser (FEL), as proposed with quantitative schemes in Refs. [6-8]. Recently, the coupling of a laserplasma accelerated electron beam to an undulator to generate synchrotron radiation has been experimentally demonstrated [9].

Laser-plasma accelerators are realized by high-intensity lasers focused onto a plasma, typically produced using a gas jet or discharge capillary $[10,11]$. The laser pulse

\footnotetext{
*Corresponding author. florian.gruener@mpq.mpg.de
}

excites large plasma waves via the ponderomotive force. The plasma wave, i.e., electron-ion charge separation, supports ultrahigh electric field gradients (as high as $\mathrm{TV} / \mathrm{m}$, several orders of magnitude above conventional accelerators) and can be used for electron acceleration. Prior to 2004, laser-plasma-accelerator experiments operated in the self-modulated regime [12-17]. Beams generated in these self-modulated laser wakefield accelerator experiments were typically characterized with large (exponential) energy spreads and bunch durations of the order of the laser pulse duration [18]. By using shorter, higher intensity lasers and extending the laser-plasma interaction length, high-quality electron bunches (percent-level relative energy spread, mrad divergences) were generated [1$3,19,20]$ with energies up to $1 \mathrm{GeV}[4,5]$. For these shortpulse, high-intensity laser experiments, an electron-free region, or bubble [21], can be generated following the laser. Background plasma electrons can become trapped in such plasma waves and accelerated over much shorter distances than in conventional accelerators (whose gradients are restricted to a few tens of $\mathrm{MV} / \mathrm{m}$ ). In the bubble regime, the longitudinal length of the beam is a fraction of the plasma wavelength $\left(<\lambda_{p} / 2\right)$. For a plasma density of $10^{19} \mathrm{~cm}^{-3}$, the plasma wavelength is approximately $10 \mu \mathrm{m}$ (and, hence, the bunch length is $\sim$ micron). The transverse beam size must be less than the laser spot size (approximately the plasma wavelength for efficient coupling), and therefore initially on the order of a few microns. During propagation in vacuum (owing to the initial beam divergence, typically $\sim 1 \mathrm{mrad}$ ), the beam transverse size 
can grow to tens of microns over centimeters of propagation.

Stability and improved beam quality can be achieved using trigger injection methods to control the injection of background plasma electrons into the plasma wave. Lasertriggered injection [22-26] is a promising method of controlling the trapping of plasma electrons, and has been successfully demonstrated using a two-pulse beating geometry $[27,28]$. Plasma density tailoring may also be employed for controlled injection [29], and negative density gradient injection has been experimentally demonstrated [30]. Further improvement may be achieved using both laser-triggered injection and density tailoring [31].

Another key feature of laser-plasma accelerators is that significant charge (up to nC) $[1,3,13]$ can be trapped in bunch lengths a fraction of the plasma wavelength, hence only a few microns for typical plasma densities. Therefore, peak currents of up to $100 \mathrm{kA}$ are realizable. Recent publications on laser-plasma-accelerator-based FELs [6] have discussed the benefits of such ultrahigh peak currents, such as drastically shrinking the length of the FEL undulator by 1 or 2 orders of magnitude, hence allowing for socalled "tabletop" FELs. On the other hand, such ultracompact electron bunches are subject to large space-charge effects. In this paper we will present a numerical and analytical study of the space-charge induced bunch expansion and energy variation. In this work we will consider parameters of electron beams produced in the bubble, or cavitated, regime of laser-plasma accelerators. Previous work on space-charge dynamics [32,33] has focused on large energy spread beams generated in the self-modulated regime of laser-plasma accelerators.

Recent publications $[34,35]$ on space-charge effects in laser-plasma-accelerators considered non-self-consistent, perturbative approaches to examine the space-charge induced energy variation along the bunch and found that the energy chirp grows linearly with propagation distance. In particular, Refs. [34,35] predicted electron energy chirps on the order of $\pm 25 \%$ for the beam parameters given in Ref. [6], i.e., for a relatively low electron energy $(150 \mathrm{MeV})$ and a high peak current $(50 \mathrm{kA})$ beam in an $80 \mathrm{~cm}$ undulator. In this work we show that these previous calculations significantly overestimate the energy chirp due to the non-self-consistent treatment applied. That, with self-consistent beam evolution included, the energy chirp reaches a maximum and decreases thereafter.

This paper is organized as follows. In Sec. II a non-selfconsistent analytical treatment of the space-charge induced energy chirp is presented. Both a diverging electron beam and a nonevolving beam distribution are considered. In Sec. III an upper limit for the amount of space-charge induced energy chirp using conservation of energy considerations is derived. Analytical expressions are reported to estimate the space-charge-driven bunch expansion time scales in Sec. IV. Section V discusses numerical results from a self-consistent point-to-point interaction simulation. The impact of space-charge-induced energy chirps on the FEL performance as well as possible solutions are discussed in Sec. VI. Conclusions are offered in Sec. VII.

\section{SPACE-CHARGE USING NON-SELF- CONSISTENT BEAM PROPAGATION}

In this section the predictions made by a non-selfconsistent, perturbative treatment of the space-charge forces are examined. The fundamental assumption made in this section is that the bunch distribution is assumed to evolve neglecting space-charge effects.

For an electron in vacuum, with charge $-e$, moving at constant relativistic velocity $v \simeq c$, with $\gamma=(1-$ $\left.v^{2} / c^{2}\right)^{-1 / 2}$, the electric fields parallel $(z)$ and perpendicu$\operatorname{lar}(r)$ to the direction of propagation are [36]

$$
\begin{aligned}
& E_{z}(\boldsymbol{x}, t)=-\operatorname{e\gamma } \zeta\left[r^{2}+\gamma^{2} \zeta^{2}\right]^{-3 / 2}, \\
& E_{r}(\boldsymbol{x}, t)=-\operatorname{e\gamma r}\left[r^{2}+\gamma^{2} \zeta^{2}\right]^{-3 / 2},
\end{aligned}
$$

where $\zeta=z-v t$ is the comoving variable. Assuming the electron is in a steady-state regime and transient effects are negligible, the Fourier transform in $\zeta$ [i.e., $\tilde{E}(k)=$ $\left.\int d \zeta \exp (i k \zeta) E(\zeta)\right]$ of Eqs. (1) and (2) may be performed (integration over all time):

$$
\begin{aligned}
& \tilde{E}_{z}(r, k)=\frac{-2 i e}{\gamma r}\left[\left(\frac{k r}{\gamma}\right) K_{0}\left(\frac{k r}{\gamma}\right)\right], \\
& \tilde{E}_{r}(r, k)=\frac{-2 e}{r}\left[\left(\frac{k r}{\gamma}\right) K_{1}\left(\frac{k r}{\gamma}\right)\right],
\end{aligned}
$$

where $K_{0}$ and $K_{1}$ are modified Bessel functions. The bunch exiting the plasma accelerator will be in a steady-state regime provided the bunch has propagated a distance greater than the formation length $\sim \gamma^{2} \sigma_{z}$ (where $\sigma_{z}$ is the characteristic longitudinal beam length, i.e., approximately the shortest coherent wavelength) after the laserplasma accelerator. Equations (1)-(4) also assume propagation in free space. The free space assumption will be valid only if there are no dielectric boundaries near the beam such that $L_{\perp} \gg \gamma \sigma_{z}$, where $\gamma \sigma_{z}$ is the characteristic transverse extent of the fields and $L_{\perp}$ is the transverse distance to the nearest boundary (e.g., beam pipe or undulator).

For a beam charge distribution $\rho(\boldsymbol{x})$, such that $\int d^{3} \boldsymbol{x} \rho=$ $-e N$, where $N$ is the total number of electrons, the total longitudinal force at $\boldsymbol{x}$ within the beam is given by

$$
F(\boldsymbol{x}, t)=\int d^{3} \boldsymbol{x}^{\prime} \rho\left(\boldsymbol{x}^{\prime}\right) E_{z}\left(\left|\boldsymbol{r}^{\prime}-\boldsymbol{r}\right|, \zeta^{\prime}-\zeta\right) .
$$

The Fourier transform of the force on axis ( $r=0)$, assuming a cylindrically symmetric beam $\rho(r, \zeta)$ propagating along the axis, is 


$$
\tilde{F}(r=0, k)=-e N \tilde{f}_{\|}(k) \int 2 \pi r^{\prime} d r^{\prime} f_{\perp}\left(r^{\prime}\right) \tilde{E}_{z}\left(r^{\prime}, k\right),
$$

with $\tilde{f}_{\|}(k)=\int d \zeta \exp (i k \zeta) f_{\|}(\zeta)$, and we have assumed $\rho=-e N f_{\perp}(r) f_{\|}(\zeta)$. This separability ansatz will be valid provided the variation in $\zeta$ of the transverse beam distribution is negligible over the length of the beam $\sigma_{z}$. For a Gaussian longitudinal distribution $f_{\|}(\zeta)=$ $\exp \left(-\zeta^{2} / 2 \sigma_{z}^{2}\right) /\left(\sqrt{2 \pi} \sigma_{z}\right)$, and $\tilde{f}_{\|}(k)=\exp \left(-k^{2} \sigma_{z}^{2} / 2\right)$. Note that, for a cylindrically symmetric beam, the electrons near the axis are dominated by the longitudinal spacecharge forces. Longitudinal versus transverse bunch expansion is discussed in Sec. III. The inverse transform (force at a beam slice $\zeta$ ) is $F(\zeta)=\int(d k / 2 \pi) \times$ $\exp (-i k \zeta) \tilde{F}(k)$, and the differential force along the beam (inducing a chirp on axis) is $d F / d \zeta=$ $-i \int(d k / 2 \pi) k \exp (-i k \zeta) \tilde{F}(k)$. For a Gaussian transverse distribution $f_{\perp}(r)=\exp \left(-r^{2} / \sigma_{r}^{2}\right) /\left(\pi \sigma_{r}^{2}\right)$, the force along the axis within the beam is

$$
\tilde{F}(r=0, k)=\frac{i e^{2} N}{k \sigma_{r}^{2}} \tilde{f}_{\|} R^{2} \exp \left(R^{2} / 4\right) \Gamma\left(0, R^{2} / 4\right),
$$

where $R=|k| \sigma_{r} / \gamma$ and $\Gamma$ is the incomplete gamma function. In the limit $R \ll 1, \Gamma\left(0, R^{2} / 4\right) \simeq 0.81-2 \ln (R)$.

Typically an electron beam exiting a laser-plasma accelerator into vacuum is emittance dominated and rapidly diverging (with rms divergence of $\sigma_{\theta} \sim \mathrm{mrad}$ ) (as discussed in Ref. [6]). It is emittance dominated in the sense that the initial beam divergence at the exit of the laserplasma accelerator is larger than the space-charge-induced transverse expansion $\sigma_{\theta}^{2}(z=0) \gg 2\left(I / I_{\mathrm{A}}\right) / \gamma^{3}$, where $I / I_{\mathrm{A}}$ is the beam current normalized to the Alfvén current. Assuming a divergence dominated beam such that $\sigma_{r}(z)=$ $\sigma_{\theta} z$, and using Eq. (7), the total energy change $\Delta \gamma(r=$ $0, \Delta z, \zeta)$ over a propagation distance $\Delta z$ is

$$
\Delta \gamma=\frac{2 i r_{e} N}{\pi \gamma^{2}} \Delta z \int d k e^{-i k \zeta} k \tilde{f}_{\|} \frac{\left[I\left(R_{1}\right)-I\left(R_{0}\right)\right]}{R_{1}-R_{0}},
$$

where $r_{e}=e^{2} / m c^{2}$ is the classical electron radius, $R_{0,1}=$ $|k| \sigma_{r}\left(z_{0,1}\right) / \gamma=|k| \sigma_{\theta} z_{0,1} / \gamma$ is the initial and final normalized beam radii, $R_{1}-R_{0}=|k| \sigma_{\theta} \Delta z / \gamma$, and $I=$ $\int d R \exp \left(R^{2} / 4\right) \Gamma\left(0, R^{2} / 4\right) / 4$. Figure 1 shows the functional dependence of $I(R)$. As Fig. 1 indicates, $\Delta \gamma$ will be significantly reduced if $R \geqslant 2 \pi$, or $\sigma_{r}>\gamma \lambda \sim \gamma \sigma_{z}$. This can be understood by considering that the characteristic transverse extent of the self-fields is $\sim \gamma \lambda$ [cf. Eqs. (3) and (4)] and the shortest coherent wavelength is $\lambda \sim \sigma_{z}$. Once the beam transverse dimensions grow larger than the extent of the Coulomb fields, the force on the beam axis is reduced. Assuming $\sigma_{r}$ is constant, $\left[I\left(R_{1}\right)-I\left(R_{0}\right)\right] /\left(R_{1}-\right.$ $\left.R_{0}\right) \rightarrow d I / d R=\exp \left(R^{2} / 4\right) \Gamma\left(0, R^{2} / 4\right) / 4$.

As we will show below, modeling the beam distribution evolution is critical to properly evaluating space-charge effects. For example, consider the experimentally demonstrated laser-plasma accelerated electron beam reported in

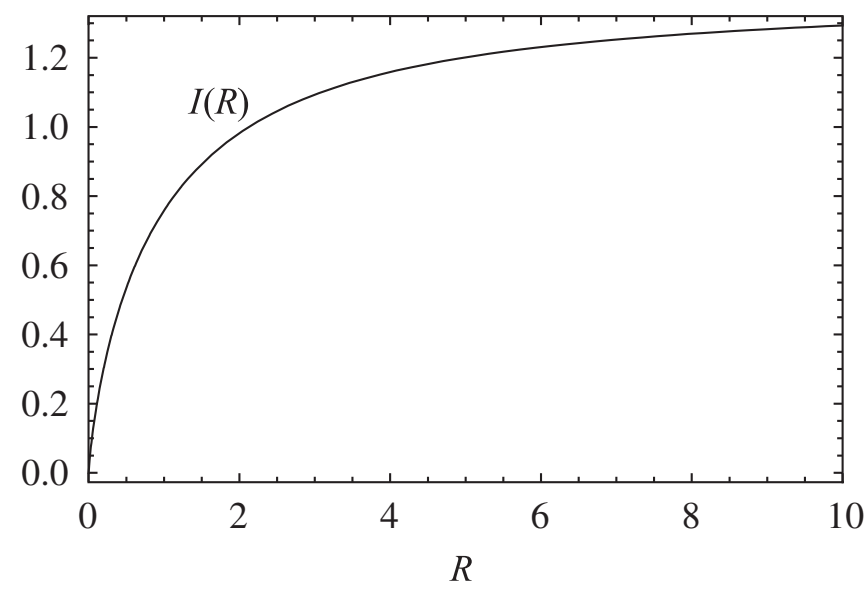

FIG. 1. Functional dependence of $I=\int d R e^{R^{2} / 4} \Gamma\left(0, R^{2} / 4\right) / 4$.

Ref. [1]. In this work, a plasma-based accelerator produced a $86 \mathrm{MeV}$ beam containing $2 \times 10^{9}$ electrons with measured energy spread of $1.8 \mathrm{MeV}$ (2\% relative energy spread), divergence of $3 \mathrm{mrad}$, and estimated peak current of $10 \mathrm{kA}$. The initial beam radius is assumed to be much less than the laser spot at the exit of the plasma channel $(24 \mu \mathrm{m})$. For this example we will consider the initial beam radius to be $\sigma_{r}=1 \mu \mathrm{m}$. The formation length is approximately $\gamma^{2} \sigma_{z} \sim 10 \mathrm{~cm}$, and the magnetic spectrometer was placed $82 \mathrm{~cm}$ from the exit of the plasma. Assuming a frozen bunch (as done in Refs. [34,35]), Eq. (8) with constant beam radius predicts a relative energy spread of $19 \%$. This is in contrast to the measured value of $2 \%$. Assuming a divergence dominated beam and applying Eq. (8) yields $1.5 \%$ relative energy spread, consistent with the measurement. In addition to transverse evolution, the longitudinal bunch evolution can have a significant influence on the space-charge effects. The longitudinal spacecharge effect will typically dominate the interaction over long propagation distances, as discussed in the following sections.

A frozen beam with fixed radius $\sigma_{r}=$ constant can be assumed provided the beam divergence over the propagation distance (typically of the order of a formation length) is much less than the beam radius $\sigma_{\theta} \gamma^{2} \sigma_{z} \ll \sigma_{r}$. For a constant radius Gaussian transverse distribution, Eq. (7) indicates the chirp along the beam axis is

$$
\frac{d \Delta \gamma}{d \zeta}=\frac{2 r_{e} N}{\pi \gamma^{2} \sigma_{z}^{3}} \Delta z \Lambda^{\prime}
$$

where

$$
\Lambda^{\prime}(\zeta)=\int d \bar{k} e^{-i \bar{k} \zeta / \sigma_{z}} \tilde{f}_{\|} e^{\bar{k}^{2} b^{2} / 4} \Gamma\left(0, \bar{k}^{2} b^{2} / 4\right) \bar{k}^{2} / 4,
$$

with $\bar{k}=k \sigma_{z}$ and $b=\sigma_{r} /\left(\gamma \sigma_{z}\right)$. Figure 2 shows $\Lambda^{\prime}=$ $d \Lambda / d\left(\zeta / \sigma_{z}\right)$ for $b=0.1, b=0.01$, and $b=0.001$. Note that, for typical parameters, $\Lambda^{\prime}$ is of order unity and is a relatively insensitive function of $b$ provided $b<1$ (i.e., the 


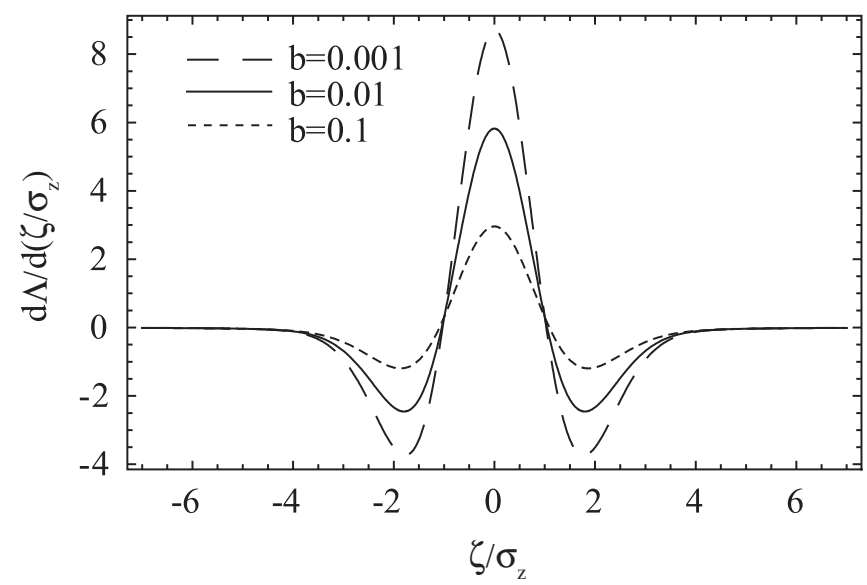

FIG. 2. The function $\Lambda^{\prime}$ [Eq. (10)] versus $\zeta / \sigma_{z}$ for $b=0.1$ (dotted curve), $b=0.01$ (solid curve), and $b=0.001$ (dashed curve).

transverse bunch size is less than the transverse extent of the Coulomb fields).

The energy change along the beam axis is $\Delta \gamma=$ $\left(2 r_{e} N / \pi \gamma^{2} \sigma_{z}^{2}\right) \Delta z \Lambda, \quad$ where $\quad \Lambda=\int^{\zeta} \Lambda^{\prime}(\tau) d\left(\tau / \sigma_{z}\right)$. Figure 3 shows $\Lambda$ and $\Lambda^{\prime}$ for $b=0.14$. For example, assuming a Gaussian bunch with $\gamma=300, \sigma_{z}=1 \mu \mathrm{m}$, $I=50 \mathrm{kA}(0.42 \mathrm{nC})$, and $\sigma_{r}=\sqrt{2} \sigma_{x}=42 \mu \mathrm{m}$, the longitudinal energy distribution along the beam axis per unit propagation distance is $m c^{2} \Delta \gamma(r=0, \zeta) / \Delta z \simeq$ $(26.5 \mathrm{MeV} / \mathrm{m}) \Lambda(\zeta)$, where $\Lambda(\zeta)$ is shown in Fig. 3. Note that Eq. (9) is the energy chirp on axis produced by a Gaussian beam, whereas Ref. [34] presents a calculation of the energy chirp assuming interacting charged disks.

One prediction of the frozen beam assumption (assumed in Refs. [34,35]) is the linear growth of the chirp with propagation distance [cf. Eq. (9)], $d \Delta \gamma / d \zeta \propto \Delta z$. This is unphysical given the finite energy in the bunch, and is a consequence of the frozen bunch assumption (i.e., assuming an infinite reservoir of potential energy). For large

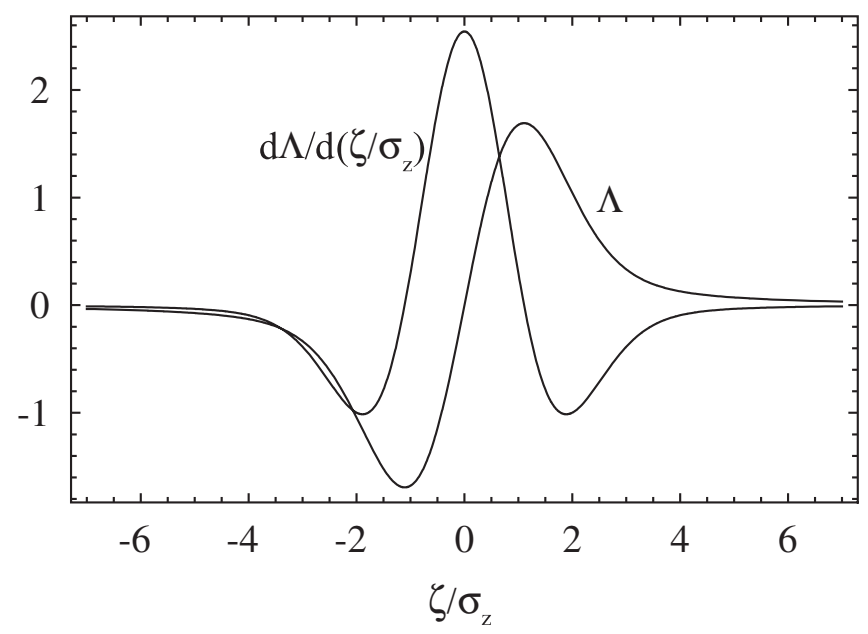

FIG. 3. The functions $\Lambda$ and $\Lambda^{\prime}$ versus $\zeta / \sigma_{z}$ for $b=0.14$. space-charge forces (or long propagation distances), the perturbative, frozen beam assumption is no longer valid and the redistribution of the bunch charge must be included to correctly model the space-charge induced energy chirp.

\section{ANALYTICAL ESTIMATE OF ENERGY CHIRP UPPER LIMIT}

If a bunch expands, its initial potential energy is transformed into kinetic energy. By energy conservation the maximum gain is reached when the bunch expansion goes to infinity. In this section we will discuss an analytical estimate for the maximal kinetic energy gain, and hence an estimate for the maximal energy chirp. In Sec. V, a selfconsistent approach is undertaken using a point-to-point particle simulation. Here we restrict ourselves to the case of a finite ensemble of discrete macroparticles, distributed as a Gaussian bunch with length $\sigma_{z}$ and transverse size $\sigma_{r}=\left(\sigma_{x}^{2}+\sigma_{y}^{2}\right)^{1 / 2}$. The number of electrons is $N$ (with total charge $Q=-N e$ ), while the number of sampling macroparticles is $M$. The introduction of macroparticles is relevant for Sec. V, as convergence studies determine a suitable value for $M$ for the particle simulations; here $M=$ $N$. In this section we assume a monoenergetic bunch with relativistic energy factor $\gamma$, hence in its rest frame the bunch length is $\sigma_{z}^{\prime}=\gamma \sigma_{z}$, while the transverse size remains $\sigma_{r}$. Therefore, the initial electrostatic potential energy in the bunch rest frame $U^{\prime}$ is

$$
U^{\prime}=e^{2}\left(\frac{N}{M}\right)^{2} \sum_{i}^{M-1} \sum_{j>i}^{M} \frac{1}{\left|\vec{r}_{i}^{\prime}-\vec{r}_{j}^{\prime}\right|} .
$$

The expectation value of $U^{\prime}$ will converge to $\sigma$ for $M \gg 1$. This value is the average inverse distance between the macroparticles, and hence just a geometrical parameter: $\sigma=\left\langle 1 / r_{i j}^{\prime}\right\rangle$. Thus, $U^{\prime}=Q^{2} \sigma$. The distance $r_{i j}^{\prime}$ between two macroparticles $i$ and $j$ is $r_{i j}^{\prime}=\left(\Delta x^{\prime 2}+\Delta y^{\prime 2}+\right.$ $\left.\Delta z^{\prime 2}\right)^{1 / 2}$, with $\Delta z^{\prime}=z_{i}^{\prime}-z_{j}^{\prime}, \Delta y^{\prime}=y_{i}^{\prime}-y_{j}^{\prime}$, and $\Delta x^{\prime}=$ $x_{i}^{\prime}-x_{j}^{\prime}$. For a Gaussian distribution $\left\langle\left(z_{i}^{\prime}-z_{j}^{\prime}\right)^{2}\right\rangle=\sigma_{z}^{\prime 2}$. To lowest order, one may hence put $\sigma \simeq 1 / \sigma_{z}^{\prime}$, if, in the rest frame, $\sigma_{z}^{\prime} \gg \sigma_{r}$. In general, there is a geometrical correction function $f$ for the contribution to $\sigma$ from the transverse direction:

$$
\left\langle 1 / r_{i j}^{\prime}\right\rangle=f\left(\sigma_{z}^{\prime} / \sigma_{r}\right) / \sigma_{z}^{\prime} .
$$

For $\quad \sigma_{z}^{\prime} \gg \sigma_{r}, \quad f \simeq \ln \left(\sigma_{z}^{\prime} / \sigma_{r}\right) / 3, \quad$ and $\quad\left\langle 1 / r_{i j}^{\prime}\right\rangle \simeq$ $\ln \left(\sigma_{z}^{\prime} / \sigma_{r}\right) /\left(3 \sigma_{z}^{\prime}\right)$. Thus, the average potential energy (normalized to $\left.m c^{2}\right)$ in the rest frame $u^{\prime}=U^{\prime} /\left(N m c^{2}\right)$ is

$$
u^{\prime} \approx \frac{I}{I_{A} \gamma} \ln \left(\gamma \sigma_{z} / \sigma_{r}\right)
$$

where $I_{A}=m c^{3} / e \simeq 17 \mathrm{kA}$ is the Alfvén current and $I=$ $Q c / \sqrt{2 \pi} \sigma_{z}$ is the peak beam current. For $\sigma_{z}^{\prime}<\sigma_{r}$ the logarithm is, approximately, replaced by a constant on the order of unity. Figure 4 shows a comparison between 
(a)

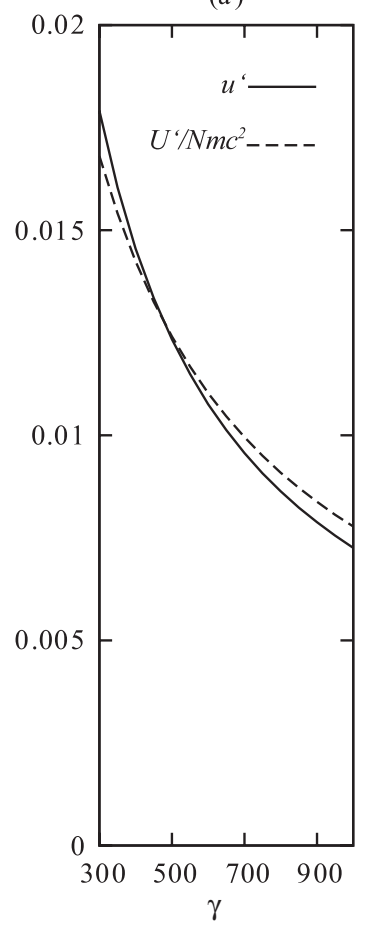

(b)

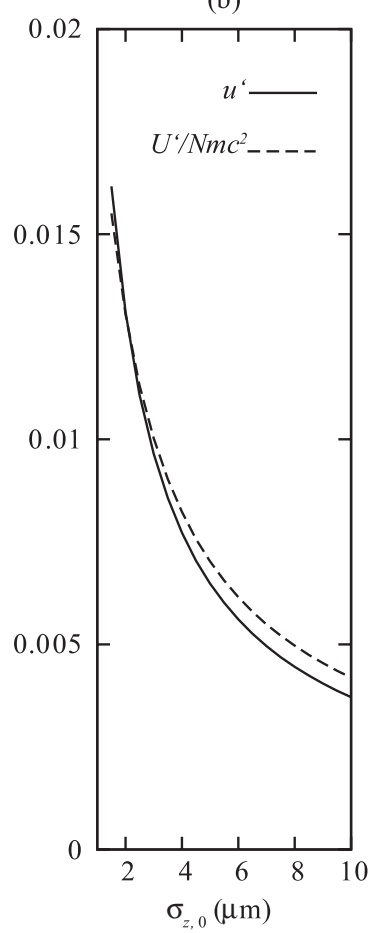

(c)

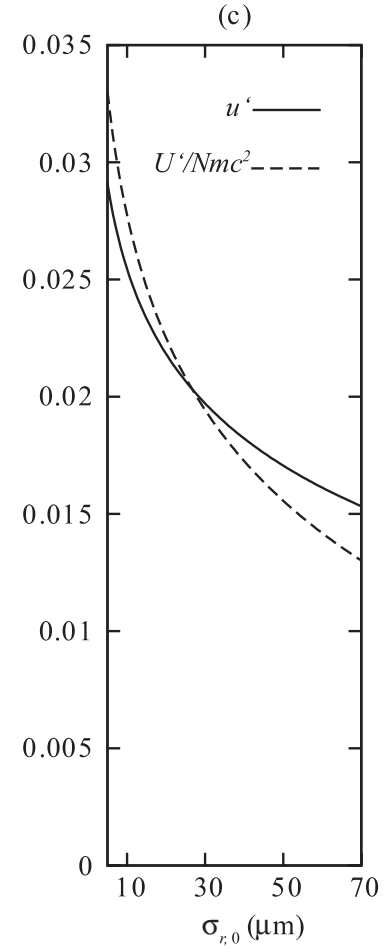

FIG. 4. Comparison between $u^{\prime}$ [Eq. (13)] (solid curves) and $U^{\prime} / N m c^{2}$ [Eq. (11)] with $M=3000$ (dashed curves) as a function of (a) energy, (b) initial longitudinal length $\sigma_{z, 0}$, and (c) initial transverse size $\sigma_{r, 0}$. Beam parameters are $Q=0.42 \mathrm{nC}, \sigma_{z, 0}=1.3 \mu \mathrm{m}$ in (a) and (c), $\sigma_{r, 0}=42 \mu \mathrm{m}$ in (a) and (b), and $\gamma=300$ in (b) and (c).

Eq. (13) and the summation Eq. (11) (normalized to $N m c^{2}$ ). For typical parameters considered here, reasonable agreement is achieved.

The dominant contribution to the average potential energy comes from the Lorentz-boosted bunch length in the bunch rest frame, $u^{\prime} \propto I / \gamma \sim Q c / \sigma_{z}^{\prime}$, whereas the average potential energy is only weakly dependent on the transverse bunch size $\sigma_{r}$. Therefore, the transverse and longitudinal bunch expansion will lead to different changes in energy. For example, to reduce $u^{\prime}$ by a factor of 2 , the bunch only needs to double its length, while for the same effect the transverse size must increase by almost an order of magnitude. Furthermore, as we will see below, the amount of (lab frame) energy change will be much larger for longitudinal bunch expansion than for transverse.

The average relativistic energy factor in the rest frame, in the limit of bunch expansion to infinity, is

$$
\gamma^{\prime}=1+u^{\prime}
$$

To estimate the maximal amplitude of the energy variation due to bunch expansion, one can consider two electrons with average energy $\gamma^{\prime}$. If these two electrons move only in the longitudinal direction in the rest frame, then their corresponding lab frame energy is (with $\beta \simeq 1$ )

$$
\gamma_{\text {long }}=\gamma^{\prime} \gamma_{0}\left(1 \pm \beta^{\prime}\right)
$$

with $\gamma_{0}$ the (initial) bunch energy and $\beta^{\prime}$ the average velocity of the two electrons in the rest frame. For the case of two electrons moving in the transverse direction only, the lab frame energy is

$$
\gamma_{\text {trans }}=\gamma^{\prime} \gamma_{0}
$$

The main finding of Eq. (15) is that, in the case of (dominant) longitudinal bunch expansion, the energy chirp in the lab frame is mainly determined by the longitudinal velocity $\beta_{z}^{\prime}$ in the rest frame. This was verified by a comparison between the longitudinal velocities $\beta_{z}^{*}$, derived from Eq. (15) whereby $\gamma_{\text {long }}$ was taken from the numerical calculation assuming a point-to-point spacecharge model (discussed in Sec. V) and $\gamma^{\prime}$ from the corresponding rest frame simulation, and the average $\beta_{z}^{\prime}$ from the rest frame simulation. The result is shown in Fig. 5. The parameters of the simulations were chosen such that the bunch expansion was dominant in the longitudinal direction according to Sec. IV. Note that the numerical calculations in each frame were done independently (the rest frame calculation using the Lorentz-boosted bunch length $\sigma_{z} \gamma_{0}$ as the initial condition). Both longitudinal velocities coincide for all energies simulated, indicating that Eq. (15) holds not just for two sample electrons, but for a full bunch simulation.

For $u^{\prime 2} \ll 1$, Eqs. (15) and (16) can be approximated as

$$
\gamma_{\text {long }} / \gamma_{0} \simeq 1+u^{\prime} \pm \sqrt{2 u^{\prime}},
$$




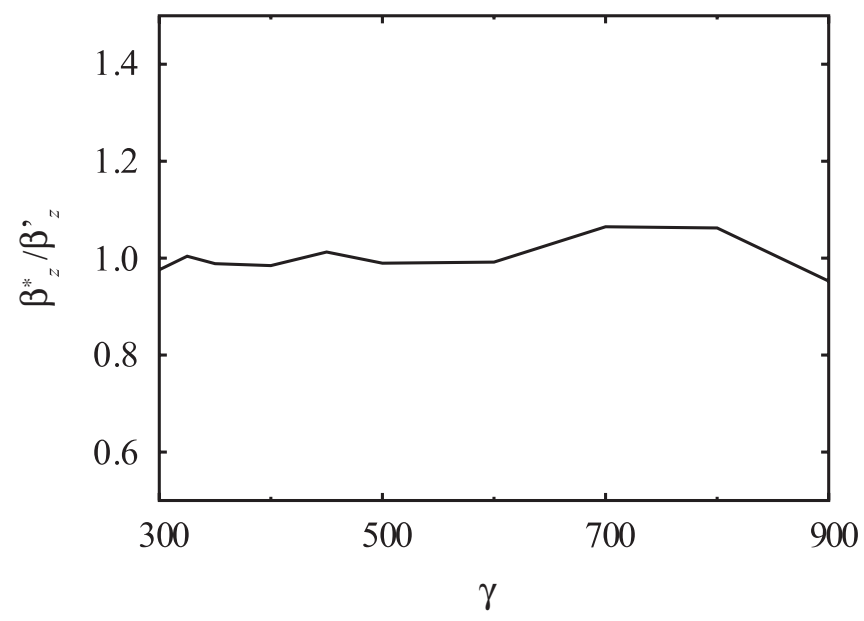

FIG. 5. The ratio of $\beta_{z}^{*}$, derived using Eq. (15) with parameters taken from the numerical calculations in both lab and rest frame runs, and $\beta_{z}^{\prime}$ from the numerical calculation in the rest frame. The parameters were chosen for the longitudinal bunch expansion regime, for which Eq. (15) must be applied.

and

$$
\gamma_{\text {trans }} / \gamma_{0}=1+u^{\prime}
$$

respectively. Bunch expansion in the longitudinal and transverse directions differ in the net energy change in the lab frame, even if in both cases the initial potential energy $u^{\prime}$ is the same. For example, if $u^{\prime}=$ 0.05 , then $\gamma_{\text {trans }} / \gamma_{0} \approx 1.05$, while $\gamma_{\text {long }-} / \gamma_{0} \approx 0.73$ and $\gamma_{\text {long }+} / \gamma_{0} \approx 1.30$; a much larger relative energy variation occurs for longitudinal bunch expansion compared to transverse expansion. In general, a purely transverse bunch expansion creates a much smaller energy variation than a longitudinal bunch expansion, because, in the longitudinalexpansion case, $u^{\prime}$ decreases with the inverse of the debunching (while in transverse case it varies logarithmically). The resulting energy chirp is larger by $\sqrt{2 u^{\prime}}$ (with $\sqrt{2 u^{\prime}} \gg u^{\prime}$, for $\left.u^{\prime} \ll 1\right)$.

\section{LONGITUDINAL AND TRANSVERSE BUNCH EXPANSION TIME SCALES}

In this section we estimate the time scales for longitudinal and transverse bunch expansion to determine which will be dominant, resulting in transverse- or longitudinalexpansion driven energy variations. Consider the plasma oscillations of a bunch with energy $\gamma$, length $\sigma_{z}$, transverse size $\sigma_{x}$, and a peak current $I$. In the case of a bunch moving in the $z$ direction at relativistic velocity, the plasma periods for transverse and longitudinal motion are different [3739]. The distance $L_{\text {long }}$ in the lab frame required for a bunch to double its length and the distance $L_{\text {trans }}$ required for doubling its transverse size are approximately

$$
L_{\text {long }}=2 \pi \gamma^{2} \sqrt{2\left(I_{A} / I\right) \sigma_{z} \sigma_{x}}
$$

and

$$
L_{\text {trans }}=2 \pi \sigma_{x} \sqrt{2\left(I_{A} / I\right) \gamma^{3}},
$$

respectively. If the transverse bunch size equals the longitudinal in the rest frame $\sigma_{x}=\gamma \sigma_{z}=\sigma_{z}^{\prime}$, then $L_{\text {long }}=$ $L_{\text {trans }}$. Therefore, the initial bunch exiting the laser-plasma accelerator, with typical dimensions $\sigma_{x} \approx \sigma_{z} \approx 1 \mu \mathrm{m}$, will predominantly undergo a transverse expansion, as discussed in Ref. [6]. It should be noted here that the transverse expansion driven by the initial emittance is stronger than that driven by Coulomb forces.

External focusing may be used to effectively keep the bunch from further transverse expansion, such that, with external focusing, the bunch will then undergo longitudinal debunching, hence developing an energy chirp approximated by Eq. (17) with an average initial energy $\gamma_{0} \approx$ $\left\langle\gamma_{\text {trans }}\right\rangle$. This prediction is verified in Sec. V with numerical calculations using a point-to-point space-charge model.

Both scalings should only be understood as estimates of the bunch expansion time scales. Typically, they differ by a factor of about 3 from the results of (self-consistent) particle calculations as discussed in the next section.

For a detailed understanding of the bunch expansion the functional dependence of bunch parameters with time is desired. The most relevant dimension is the bunch length, which is difficult to control externally (while the transverse expansion is typically controlled via external focusing). Consider the exact solution for two (nonrelativistic) electrons starting at time $t=0$ at an initial distance $L_{0}$, moving under the Coulomb force $e^{2} / L^{2}$, where $L$ is the distance between the electrons. Note that typical electron velocities in the bunch rest frame for the cases discussed here are indeed nonrelativistic. A distance $L>L_{0}$ is reached after time

$$
t=t_{0}\left\{x^{1 / 2}(x-1)^{1 / 2}+\ln \left[x^{1 / 2}+(x-1)^{1 / 2}\right]\right\},
$$

with $x=L / L_{0}$ and $t_{0}=\sqrt{m L_{0}^{3} / 4 e^{2}}$. For large $t>t_{0}, x$ scales approximately linearly, while in the initial stage for $t<t_{0}$ the "debunching" has not developed yet. This result is important for the interpretation of the temporal behavior of the energy chirps found in the particle simulations, as discussed in the following section. Note that $t_{0}$ yields the (rest frame) time scale for the onset of linear growth of $x$, while Eqs. (19) and (20) give the (lab frame) time scales for doubling the bunch length.

\section{SELF-CONSISTENT POINT-TO-POINT SIMULATIONS}

In order to study the self-consistent bunch evolution (i.e., a treatment where the space-charge fields modify the bunch distribution, which drives the space-charge force), we have used the code GENERAL PARTICLE TRACER (GPT) in a point-to-point particle interaction 
mode [40]. Point-to-point interaction models have previously been applied to study space-charge effects in large energy spread beams [33]. GPT calculates the effect of macroparticle $j$ on particle $i$ in the rest frame of particle $j$, where an electrostatic field, but no magnetic field, is produced at the position of $i$. To calculate the fields on $i$ in the $l a b$ frame of particle $j$, the corresponding Lorentz transformations are performed. This method is especially suitable for large energy spreads, as it does not rely on the definition of a bunch rest frame, but moves into the individual rest frame of each (macro)electron. The point-topoint interaction algorithm approximates retardation effects, as discussed in Ref. [33]. It, however, does assume an "eternal" bunch, i.e., a bunch that existed at least as long as all (initial) fields could propagate through the entire bunch (approximately a formation length). In this sense, GPT yields an overestimate of the space-charge force for bunches which have not propagated a formation length after the accelerator, because retardation will decrease the space-charge effect. On the other hand, from Eq. (13), we expect only a small correction due to retardation following a formation length after exiting the laser-plasma accelerator, because the self-energy scales only with the current, which is a local quantity. In the numerical pointto-point calculations below we have used $M=3 \times 10^{3}$ macroparticles (as well as convergence studies with $M=$ $\left.5 \times 10^{4}\right)$.

\section{A. Comparison between self-consistent solution and frozen bunch approximation}

In the following we use GPT to model a Gaussian electron bunch having the parameters: $Q=0.42 \mathrm{nC}, \sigma_{z}=$ $1 \mu \mathrm{m}, \gamma_{0}=300, \sigma_{x}=30 \mu \mathrm{m}, I=50 \mathrm{kA}$, and zero emittance. Here we neglect the expansion of the bunch from the exit of the laser-plasma accelerator to the undulator, i.e., the initial conditions for the simulation assume a bunch with transverse size $\sigma_{x}=30 \mu \mathrm{m}$. The initial transverse beam size exiting the laser-plasma accelerator is expected to be on the order of a few microns. GPT simulations with more realistic initial beam spot sizes, and with external focusing between the accelerator and undulator, will be discussed in Sec. V B. Here the GPT simulations were made without any focusing devices, hence the bunch is allowed to freely expand in the transverse direction. For this case, we directly compare the self-consistent evolution with the results using the frozen beam assumption (as used in Sec. II and Refs. [34,35]). Moreover, according to Eq. (18), a moderate increase in transverse size has little effect on the induced energy variation. For comparing with the results derived using a frozen beam assumption, we are mainly interested in longitudinal debunching, which has the largest effect on the induced energy spread.

Figure 6 shows the energy chirps $\langle\gamma\rangle^{-1} d\langle\gamma\rangle / d \zeta$ (at the beam centroid) versus propagation distance $z$ for different electron bunch energies as calculated with GPT. As Fig. 6
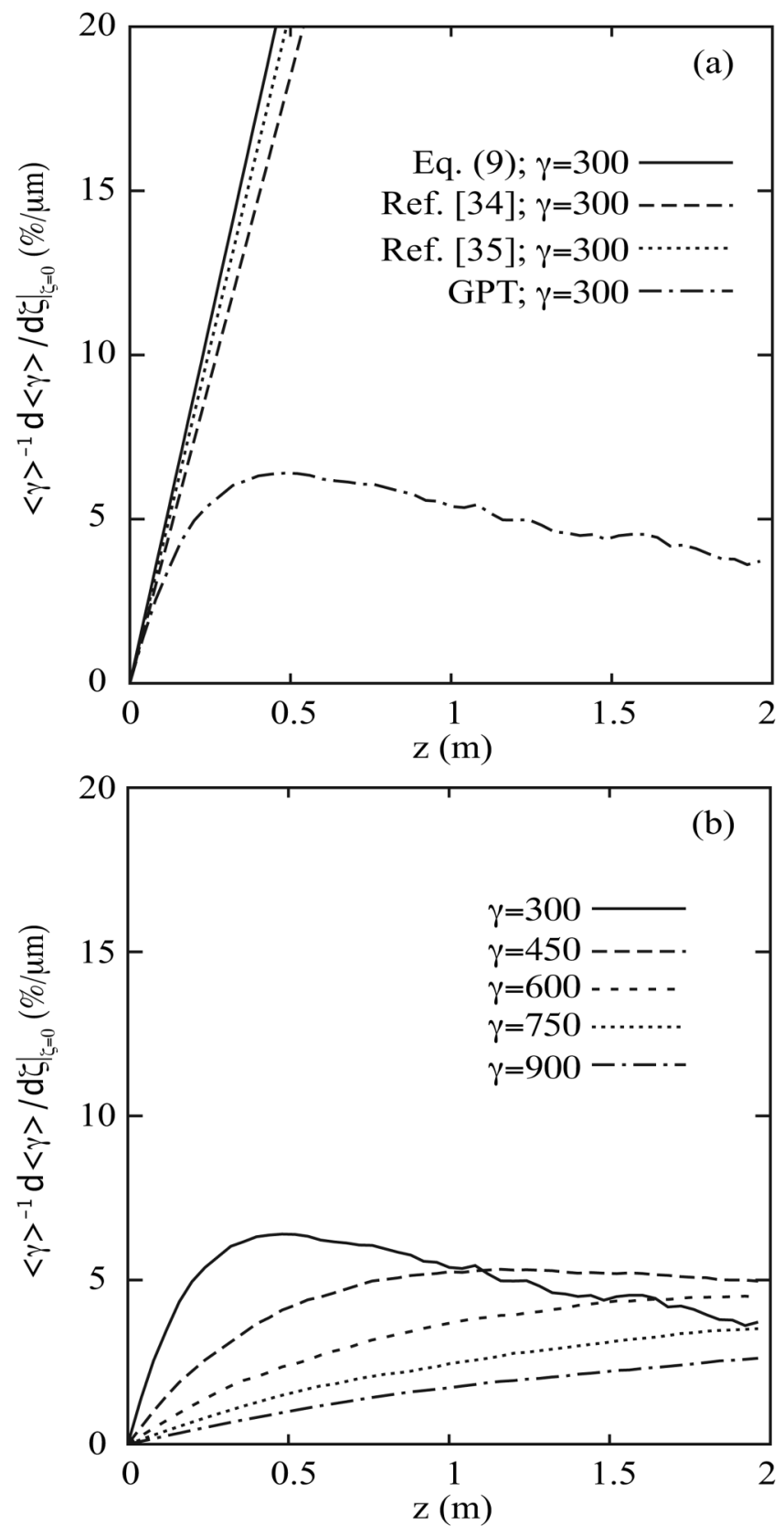

FIG. 6. Beam energy chirps $\langle\gamma\rangle^{-1} d\langle\gamma\rangle / d \zeta$ (relative chirp per micron) at the beam centroid $(\zeta=0)$ versus $z$ for different electron bunch energies calculated using GPT. The beam parameters were $\sigma_{z}=1 \mu \mathrm{m}, \sigma_{x}=30 \mu \mathrm{m}$, and $I=50 \mathrm{kA}$. (a) The chirp predicted by GPT (dash-dotted curve), the frozen beam approximation with $\gamma=300$ for a Gaussian bunch Eq. (9) (solid line), the results of Ref. [35] (dotted line), and Ref. [34] (dashed line). (b) Self-consistent GPT simulations of the spacecharge induced chirp assuming $\gamma=300,450,600,750$, and 900 versus propagation distance.

indicates, the chirps depart from an initial linear increase with propagation distance and tend towards a plateau. For the $\gamma=300$ case, the chirp declines after about $0.5 \mathrm{~m}$ of propagation. This was also the case for the higher beam 
energy cases, but occurred at greater distances. The existence of a plateau and the subsequent decline can be readily understood from the simple analytical results discussed above. The amplitude of the linear energy chirp in the lab frame is mostly determined by the longitudinal velocity in the bunch rest frame. The energy chirp is determined effectively by the ratio of energy change and bunch length, hence (in the rest frame view) by the longitudinal velocity and longitudinal bunch size. In the beginning of the Coulomb explosion, the velocity increases much faster than the debunching, leading to a linear increase of the energy chirp. At a later point, the velocity increase diminishes, while the debunching continues roughly linearly, as predicted by Eq. (21). Hence, the energy chirp in the lab frame will reach a plateau, where the growth in longitudinal velocity and debunching compensate each other. The following decline in chirp is a result of the ongoing growth in bunch length at almost constant debunching rate. The linear growth in chirp (as predicted by the non-selfconsistent analysis) fails owing to the assumption of no bunch distribution evolution.

One can also see that the larger the electron energy, the lower the energy chirp. This also agrees with the energy dependence studied above in the analytical treatments. For the initial stage, where debunching is not strong, the energy chirp is only determined by the amplitude of the energy change, where the self-energy scales inversely with the electron energy.

We have also included in Fig. 6(a) the linear increase of the on axis energy chirp for a Gaussian bunch, with $\gamma=$ 300 , predicted by the frozen beam approximation, Eq. (9). In addition, the predictions of Ref. [35] (assuming the frozen beam approximation and a flattop transverse distribution) and Ref. [34] (assuming the frozen beam approximation and modeling interacting charged disks) are shown in Fig. 6(a). The disagreement between the self-consistent evolution and the frozen beam calculation occurs after a propagation distance of about $0.2 \mathrm{~m}$ for the parameters considered. Note that this distance is only about one-fourth of the FEL saturation length for the parameters of Ref. [6] (where space-charge induced chirps were neglected), hence a self-consistent calculation must be performed.

Debunching plays a major role in the development of the energy chirp for moderate energy beams. Figure 7 shows the ratio of the bunch length at $z=1 \mathrm{~m}$ and the initial length $\sigma_{z, 0}=1 \mu \mathrm{m}$, as a function of electron energy. For lower electron beam energies, the bunch length increases by a few tens of percent, indicating a significant decrease of the peak current, while at higher energies the reduction in peak current is a few percent. This reduction in peak current will be discussed in Sec. VI.

\section{B. Beam propagation from accelerator to undulator}

Before discussing the impact of the space-charge induced energy chirps on the FEL process in Sec. VI, we

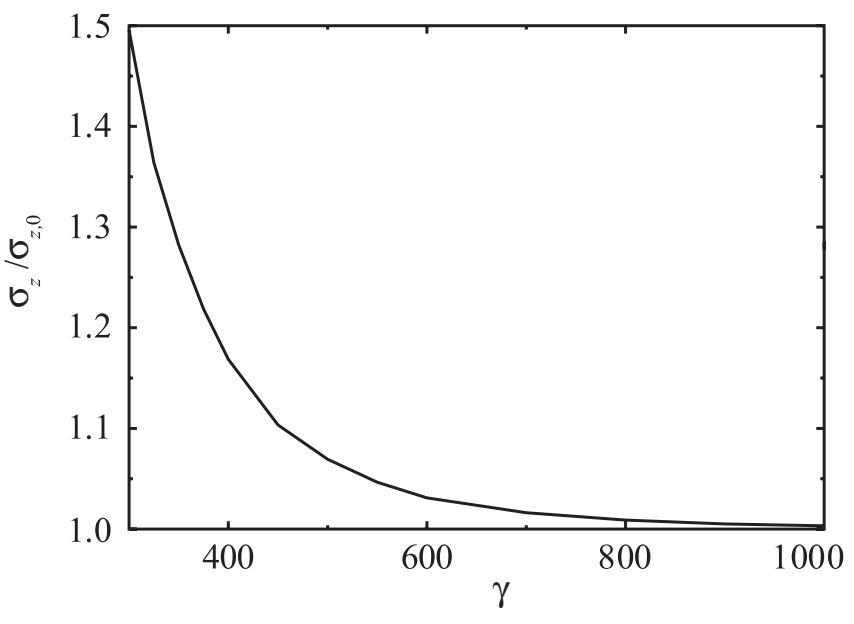

FIG. 7. Debunching $\sigma_{z}(z=1 \mathrm{~m}) / \sigma_{z}(z=0)$ as a function of electron energy for the beam parameters: $\sigma_{z}=1 \mu \mathrm{m}, \sigma_{x}=$ $30 \mu \mathrm{m}$, and $I=50 \mathrm{kA}$.

consider a more realistic experimental setup for a tabletop FEL, including the bunch transport between the exit of the laser-plasma accelerator and the undulator using external focusing devices. Here we use GPT to model a Gaussian electron bunch having the initial parameters: $Q=$ $0.42 \mathrm{nC}, \sigma_{z}=1 \mu \mathrm{m}, \sigma_{x}=1 \mu \mathrm{m}, m c^{2} \gamma=150 \mathrm{MeV}$, and $I=50 \mathrm{kA}$.

At the exit of the laser-plasma accelerator, one can assume that $\sigma_{x} \sim \sigma_{z} \sim 1 \mu \mathrm{m}$, as found in particle-in-cell simulations [41]. This initial bunch geometry together with Eqs. (19) and (20) indicate that the space-charge force is predominantly in the transverse direction. But, transverse beam evolution is typically emittance dominated in this regime (with normalized transverse emittance typically of $1 \mu \mathrm{m}$ ) [6]. Figure 8 shows the energy distribution of the bunch, assuming an initial radius exiting the plasma accelerator of $\sigma_{x, 0}=1 \mu \mathrm{m}$, after $6 \mathrm{~cm}$ and after $1.3 \mathrm{~m}$. Here we have included an external focusing optic at $8 \mathrm{~cm}$, keeping the beam spot size approximately $100 \mu \mathrm{m}$ for the considered propagation distance. The energy distribution generated, consistent with Eq. (16), is shown in Fig. 8(a), indicating a predominantly transverse expansion. The energy distribution in Fig. 8(a) is due to the Gaussian electron density distribution, where the highest density implies the largest (local) potential energy and, hence, the largest gain in kinetic energy. Note that in the case of transverse spacecharge evolution, all the beam electrons gain kinetic energy. After the focusing device (at $8 \mathrm{~cm}$ ) a quasilinear energy chirp evolves, as seen in Fig. 8(b). The focusing device prevents further transverse expansion, such that the bunch undergoes effectively only a longitudinal expansion, resulting in a linear chirp, consistent with Eq. (15). This chirp is generated about the average energy of the bunch $\langle\gamma\rangle$ that was reached after the transverse expansion. A modest increase in transverse beam size, or a zero increase 

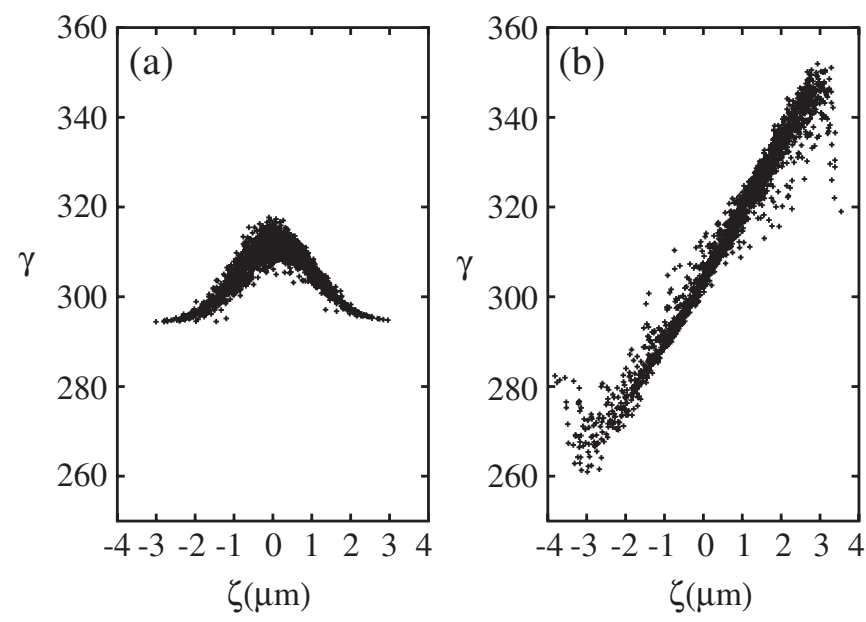

FIG. 8. Electron beam energy distribution at (a) $z=6 \mathrm{~cm}$ and (b) $z=1.3 \mathrm{~m}$ after the laser-plasma accelerator. The initial beam parameters at the exit of the laser-plasma accelerator were $\sigma_{z}=1 \mu \mathrm{m}, \sigma_{x}=1 \mu \mathrm{m}, \gamma m c^{2}=150 \mathrm{MeV}$, and $I=$ $50 \mathrm{kA}$. A focusing optic is placed at $z=8 \mathrm{~cm}$.

in the case with external focusing, implies effectively only longitudinal debunching.

The simple analytical expressions discussed in Sec. III can be used to estimate the energy gain shown in Fig. 8. If a bunch expands from an initial geometry with $\sigma_{x, 0}$ and $\sigma_{z, 0}$ and reaches after some time $t$ a new geometry $\sigma_{x, t}$ and $\sigma_{z, t}$, assuming the bunch distribution remains Gaussian, then the gain in energy can be estimated simply by the difference in potential energy $\Delta \gamma^{\prime}=-\Delta u^{\prime}=u^{\prime}(0)-u^{\prime}(t)$. For the case shown in Fig. 8(a), Eqs. (13) and (18), predict $\langle\gamma(t)\rangle /\langle\gamma(0)\rangle=1.018$, whereas GPT numerically calculates $\langle\gamma(t)\rangle /\langle\gamma(0)\rangle=1.025$.

\section{IMPACT ON FEL PERFORMANCE AND POSSIBLE SOLUTIONS}

A large energy variation along the bunch implies that the radiation emitted by electrons will not be in resonance with electrons further ahead in the beam, if the energy chirp is larger than the FEL bandwidth. The FEL resonance wavelength is

$$
\lambda=\frac{\lambda_{u}}{2 \gamma^{2}}\left(1+\frac{K^{2}}{2}\right)
$$

with $\lambda_{u}$ the undulator period and $K$ the undulator parameter $\left(K=0.93 \lambda_{u}[\mathrm{~cm}] B_{0}[\mathrm{~T}]\right.$ and $B_{0}$ the magnetic field strength on the undulator axis). The FEL bandwidth is determined by the FEL parameter [42]

$$
\rho=\frac{1}{2 \gamma}\left[\frac{I}{I_{A}}\left(\frac{\lambda_{u} A_{u}}{2 \pi \sigma_{x}}\right)^{2}\right]^{1 / 3},
$$

with $A_{u}=a_{u}\left[J_{0}(\zeta)-J_{1}(\zeta)\right]$ (planar undulator), $a_{u}^{2}=$ $K^{2} / 2, \zeta=a_{u}^{2} /\left[2\left(1+a_{u}^{2}\right)\right]$, and $J_{m}$ are Bessel functions.
Electrons within the beam can interact over a cooperation length $L_{c}=\lambda L_{\text {gain }} / \lambda_{u}$, i.e., the slippage length, between the phase of the light relative to the electron phase, over a gain length of propagation in the undulator. The gain length $L_{\text {gain }}$, or radiation power e-folding length, in the ideal, one-dimensional case (without energy spread, emittance, and diffraction effects) is given by

$$
L_{\text {gain }}=\frac{\lambda_{u}}{4 \pi \sqrt{3} \rho}
$$

and the cooperation length is $L_{c}=\lambda /(4 \pi \sqrt{3} \rho)$.

Efficient FEL performance requires the relative chirp over a cooperation length be less than the FEL parameter:

$$
\frac{L_{c}}{\gamma} \frac{d \gamma}{d \zeta}<\rho
$$

For varying beam charge and energy, the FEL parameter scales as $\rho \propto I^{1 / 3} / \gamma$, and, assuming the frozen beam approximation Eq. (9), the chirp over a cooperation length scales as $\left(L_{c} / \gamma\right)(d \gamma / d \zeta) \propto I^{2 / 3} / \gamma^{4}$, such that, for sufficiently high current and low energy, the space-charge fields may affect the FEL process.

Consider the FEL and beam parameters [6]: $\lambda=32 \mathrm{~nm}$, $\lambda_{u}=5 \mathrm{~mm}, \rho=0.01, \quad \sigma_{z}=1 \mu \mathrm{m}, \quad \sigma_{x}=30 \mu \mathrm{m}$, $\gamma m c^{2}=150 \mathrm{MeV}$, and $I=50 \mathrm{kA}$. The frozen bunch approximation Eq. (9) predicts $\left(L_{c} / \rho \gamma\right) d \gamma / d \zeta \simeq 5$ at $z=$ $80 \mathrm{~cm}$. The self-consistent numerical calculation, including bunch evolution (cf. Fig. 6) predicts $\left(L_{c} / \rho \gamma\right) d \gamma / d \zeta=$ 1.1. Note that, as Fig. 6 shows, the energy chirp computed self-consistently does not increase linearly with propagation distance as predicted using the frozen beam approximation (used in Refs. [34,35]).

As mentioned above, longitudinal debunching implies a reduction in peak current. This effect is presently not included in standard FEL codes used to model the FEL radiation generation, e.g., GENESIS [43], and, therefore, extensions of GENESIS in order to implement a timedependent current distribution must be considered to model the FEL in this regime [44]. The FEL code GENESIS includes space-charge effects, however, only the short-range space-charge working against microbunching. The effects of the long-range space-charge fields discussed in this work are equivalent to induced resistive wall wakefields. (The effect of resistive wall wakefields, for the parameters in the above example, are discussed in Ref. [6].) Therefore, the impact of space-charge on beam energy spread can be modeled using GENESIS, but with longitudinal debunching excluded. The debunching is less severe at higher beam energies, as depicted in Fig. 7, where the reduction in peak current is of the order of a few percent.

If the energy chirp is on the order of the FEL parameter, one can consider several possible solutions to mitigate the 
effect of the chirp. The simplest option is to operate the FEL (at the same desired wavelength) with higher energy electrons. As shown in Fig. 6, going to higher beam energies significantly reduces the chirp, as well as the decrease in peak current (Fig. 7).

One solution to mitigate the effects of the space-charge induced energy chirp is to operate the laser-plasma accelerator such that a negative energy chirp on the electron beam (with head lower energy than tail) is generated upon exit of the accelerator into vacuum. This can be accomplished by varying the plasma density and accelerator length such that the beam exits the plasma accelerator past the dephasing length. The space-charge forces will then act to remove this negative chirp from the beam, allowing the electrons to be at the resonant energy in the undulator.

An additional option for reducing the effect of an energy chirp is to maintain the resonance condition by tapering, i.e., by making the undulator parameter dependent on the position along the undulator, such that the resonance condition is always fulfilled. Because of phase slippage, the energy of the beam, at a phase of the light, varies with propagation distance $\gamma=\gamma(z)$, and, from Eq. (22), by varying the undulator parameter $K=K(z)$, the resonant condition can be maintained $\lambda(z)=\lambda(z=0)$. Spacecharge induced energy chirps imply that $\gamma(z)$ is an increasing function with $z$ and, hence, $K(z)$ must also increase. In practice, the use of tapering is limited because the variation in $K$ has an upper limit. Typically $K$ is increased by reducing the undulator gap, which has a natural minimum. Furthermore, tapering will only be effective for a certain portion of the bunch, whereas the rest of the electrons move out of resonance.

\section{CONCLUSIONS}

In this paper we have examined energy variations along an electron bunch induced by space-charge effects. Spacecharge effects can play a significant role in bunches generated by laser-plasma accelerators due to their ultrahigh peak currents (typically an order of magnitude greater than conventional accelerators). Using point-to-point particle simulations we have seen that self-consistent bunch redistribution, and, in particular, longitudinal debunching, causes the beam energy chirp to depart from the linear increase predicted by the frozen beam approximation (described in Sec. II and used in, e.g., Refs. [34,35]). For sufficiently long propagation distances the energy chirp reaches a plateau, and then declines. The plateau is reached when the space-charge induced growth in longitudinal velocity (in the bunch rest frame) is compensated by the increasing bunch length. Since the impact of large chirps on the FEL process can be significant, there is a minimum electron energy at which a tabletop FEL could operate, including chirp compensation by tapering, for a given set of beam and undulator parameters.

\section{ACKNOWLEDGMENTS}

We are grateful for constructive discussions with E. Esarey, A. Meseck, M. Dohlus, T. Limberg, S. Reiche, and W. Leemans. This work has been funded by DFG through Transregio TR18 and supported by the DFG cluster-of-excellence Munich Center for Advanced Photonics MAP. Work at LBNL was supported by the Director, Office of Science, of the U.S. Department of Energy under Contract No. DE-AC02-05CH11231.

[1] C. G. R. Geddes, C. Toth, J. van Tilborg, E. Esarey, C. B. Schroeder, D. Bruhwiler, C. Nieter, J. Cary, and W. P. Leemans, Nature (London) 431, 538 (2004).

[2] S. P. D. Mangles et al., Nature (London) 431, 535 (2004).

[3] J. Faure, Y. Glinec, A. Pukhov, S. Kiselev, S. Gordienko, E. Lefebvre, J.-P. Rousseau, F. Burgy, and V. Malka, Nature (London) 431, 541 (2004).

[4] W. P. Leemans, B. Nagler, A. J. Gonsalves, C. Tóth, K. Nakamura, C. G. R. Geddes, E. Esarey, C. B. Schroeder, and S. M. Hooker, Nature Phys. 2, 696 (2006).

[5] K. Nakamura, B. Nagler, C. Tóth, C. G. R. Geddes, C. B. Schroeder, E. Esarey, W. P. Leemans, A. J. Gonsalves, and S. M. Hooker, Phys. Plasmas 14, 056708 (2007).

[6] F. Grüner et al., Appl. Phys. B 86, 431 (2007).

[7] C.B. Schroeder, W. M. Fawley, E. Esarey, and W. P. Leemans, in Proceedings of the 28th International Free Electron Laser Conference, Berlin, Germany, 2006, p. 455, http://www.JACoW.com.

[8] K. Nakajima, Nature Phys. 4, 92 (2008).

[9] H.-P. Schlenvoigt et al., Nature Phys. 4, 130 (2008).

[10] D. J. Spence and S. M. Hooker, Phys. Rev. E 63, 015401 (2000).

[11] A. Butler, D. J. Spence, and S. M. Hooker, Phys. Rev. Lett. 89, 185003 (2002).

[12] W. P. Leemans, P. Catravas, E. Esarey, C. G. R. Geddes, C. Toth, R. Trines, C. B. Schroeder, B. A. Shadwick, J. van Tilborg, and J. Faure, Phys. Rev. Lett. 89, 174802 (2002).

[13] V. Malka et al., Science 298, 1596 (2002).

[14] A. Modena et al., Nature (London) 377, 606 (1995).

[15] K. Nakajima et al., Phys. Rev. Lett. 74, 4428 (1995).

[16] D. Umstadter, S.-Y. Chen, A. Maksimchuk, G. Mourou, and R. Wagner, Science 273, 472 (1996).

[17] A. Ting, C. I. Moore, K. Krushelnick, C. Manka, E. Esarey, P. Sprangle, R. Hubbard, H. R. Burris, R. Fischer, and M. Baine, Phys. Plasmas 4, 1889 (1997).

[18] J. van Tilborg, C. B. Schroeder, C. V. Filip, C. Tóth, C. G. R. Geddes, G. Fubiani, R. Huber, R. A. Kaindl, E. Esarey, and W.P. Leemans, Phys. Rev. Lett. 96, 014801 (2006).

[19] S. Karsch et al., New J. Phys. 9, 415 (2007).

[20] J. Osterhoff et al., Phys. Rev. Lett. 101, 085002 (2008).

[21] A. Pukhov and J. Meyer-ter-Vehn, Appl. Phys. B 74, 355 (2002).

[22] D. Umstadter, J. K. Kim, and E. Dodd, Phys. Rev. Lett. 76, 2073 (1996).

[23] E. Esarey, R. F. Hubbard, W. P. Leemans, A. Ting, and P. Sprangle, Phys. Rev. Lett. 79, 2682 (1997). 
[24] C. B. Schroeder, P. B. Lee, J. S. Wurtele, E. Esarey, and W. P. Leemans, Phys. Rev. E 59, 6037 (1999).

[25] H. Kotaki, S. Masuda, M. Kando, J. K. Koga, and K. Nakajima, Phys. Plasmas 11, 3296 (2004).

[26] G. Fubiani, E. Esarey, C. B. Schroeder, and W. P. Leemans, Phys. Rev. E 70, 016402 (2004).

[27] J. Faure, C. Rechatin, A. Norlin, A. Lifschitz, Y. Glinec, and V. Malka, Nature (London) 444, 737 (2006).

[28] H. Kotaki et al., IEEE Trans. Plasma Sci. 36, 1760 (2008).

[29] S. Bulanov, N. Naumova, F. Pegoraro, and J. Sakai, Phys. Rev. E 58, R5257 (1998).

[30] C. G. R. Geddes, K. Nakamura, G. R. Plateau, C. Toth, E. Cormier-Michel, E. Esarey, C. B. Schroeder, J. R. Cary, and W. P. Leemans, Phys. Rev. Lett. 100, 215004 (2008).

[31] G. Fubiani, E. Esarey, C. B. Schroeder, and W. P. Leemans, Phys. Rev. E 73, 026402 (2006).

[32] A. W. Chao, R. Pitthan, T. Tajima, and D. Yeremian, Phys. Rev. ST Accel. Beams 6, 024201 (2003).

[33] G. Fubiani, J. Qiang, E. Esarey, W. P. Leemans, and G. Dugan, Phys. Rev. ST Accel. Beams 9, 064402 (2006).

[34] G. Geloni, E. Saldin, E. Schneidmiller, and M. Yurkov, Nucl. Instrum. Methods Phys. Res., Sect. A 578, 34
(2007).

[35] R. A. Bosch, Phys. Rev. ST Accel. Beams 10, 050701 (2007).

[36] J.D. Jackson, Classical Electrodynamics (Wiley, New York, 1975).

[37] J. Rosenzweig, C. Pellegrini, L. Serafini, C. Ternieden, and G. Travish, Nucl. Instrum. Methods Phys. Res., Sect. A 393, 376 (1997).

[38] L. Serafini and J. B. Rosenzweig, Phys. Rev. E 55, 7565 (1997).

[39] M. Ferrario, in The Physics and Applications of High Brightness Electron Beams, edited by J. Rosenzweig, G. Travish, and L. Serafini (World Scientific, Singapore, 2003).

[40] http://www.pulsar.nl/gpt.

[41] M. Geissler, J. Schreiber, and J. Meyer-ter-Vehn, New J. Phys. 8, 186 (2006).

[42] R. Bonifacio, C. Pellegrini, and L. M. Narducci, Opt. Commun. 50, 373 (1984).

[43] S. Reiche, Nucl. Instrum. Methods Phys. Res., Sect. A 429, 243 (1999).

[44] S. Reiche (private communication). 